\title{
La terapia a nanoescala: ensamblaje de estructuras liberadoras de fármacos
}

\author{
Sergio Alcalá-Alcalá, * David Quintanar-Guerrero *
}

\begin{abstract}
RESUMEN: La nanotecnología es una ciencia que posee grandes expectativas en diversas áreas, pues contempla la obtención de bloques de materia muy pequeños, creados a nivel molecular o atómico, para el desarrollo de nuevos dispositivos o materiales con propiedades mejoradas. Una de las aplicaciones más prometedoras de la nanotecnología es la nanomedicina, la cual involucra el desarrollo de nanosistemas diseñados para monitorear la salud, liberar fármacos, curar enfermedades o reparar tejidos. Respecto a la liberación de fármacos, ésta se ha asentado en el antiguo concepto de la "bala mágica", en el que se establece una búsqueda de diminutas partículas o nanoacarreadores capaces de dirigir sustancias activas al sitio donde son requeridas, con el objetivo de mantener y controlar la entrega del fármaco en tiempo y espacio. La intensa investigación de las últimas tres décadas ha demostrado que los nanoacarreadores cumplen con esas expectativas y representan una mejor respuesta para atender las necesidades terapéuticas actuales.
\end{abstract}

PALABRAS CLAVE: nanotecnología, nanopartículas, nanomedicina, nanoacarreadores, liposomas

ABSTRACT: Nanotechnology is a science that has great expectations in several areas, because it includes the obtaining of very small blocks of matter, created a molecular or atomic level, to develop new devices or materials with improved properties. One of the most promising applications of nanotechnology is nanomedicine, which involves the development of nano-systems designed to monitor health, deliver drugs, cure diseases and/or repair tissues. Regarding drug delivery, this has been based on the old concept of the "magic bullet", in which is established a research of tiny particles or nanocarriers that are able to lead drug to the site where they are required, in order to maintain and control the drug release in time and space.

KEYWORDS: nanotechnology, nanoparticles, nanomedicine, nanocarriers, liposomes

\section{Introducción-nanotecnología}

La nanotecnología es una ciencia que nace en la década de los años sesenta del siglo XX cuando Feynman propone la idea de conocer y sobre todo manipular o controlar la materia en la escala atómica o molecular con la finalidad de aplicar este conocimiento en diferentes áreas de la tecnología como la óptica, la química, la eléctrica y la medicina. Con base en la National Nanotechnology Initative (NNI), podemos definir la nanotecnología como el área encargada del estudio, diseño, caracterización, síntesis y aplicación de

\footnotetext{
* Laboratorio de Investigación y Posgrado en Tecnología Farmacéutica, Facultad de Estudios Superiores Cuautitlán, Universidad Nacional Autónoma de México. Avenida $1^{\circ}$ de Mayo s/n, Campo 1, Cuautitlán Izcalli, Estado de México, C.P. 54743. México. Tel. (152) 55 56232065.

Correspondencia: (quintana@servidor.unam.mx).
} 
materiales y sistemas, cuyas estructuras y componentes exhiben nuevas y mejoradas propiedades físicas, químicas o biológicas debido a su tamaño nanométrico $\left(1 \mathrm{~nm}=10^{-9} \mathrm{~m}\right)$ [1]. Estas nuevas propiedades ganan importancia debido al incremento del área superficial; de tal manera que los efectos cuánticos y las propiedades de superficie adquieren significancia, haciendo posible la manipulación de los materiales a nivel atómico, molecular o supremolecular con diferentes intenciones u objetivos [2].

Se han descrito dos enfoques para abordar la nanotecnología el bottomup o construcción de abajo hacia arriba y el top-down o construcción de arriba hacia abajo. En el proceso bottom-up, los átomos y moléculas son ensamblados uno a uno, en reacciones químicas controladas, como bloques de construcción que van creciendo hasta generar la nanoestructura deseada. Este autoensamblaje de átomos o moléculas puede llevarse a cabo incluso empleando una plantilla (como, por ejemplo, biomacromoléculas, micelas poliméricas, copolímeros o surfactantes no iónicos) que funge como plataforma de interacción, donde una secuencia específica, patrón o forma permiten ir diseñando la nanoestructura. Por otro lado, el enfoque top-down utiliza técnicas como la fragmentación, corte o grabado, a través de procesos de mecanizado de película, de superficie o litografía (de rayos $\mathrm{x}$, de microelectro-sistemas, de haz de electrones, la fotolitografía) para reducir la talla de los materiales en bulto hasta la escala nanométrica. El enfoque bottom-up se considera ideal para ser aplicado en nanotecnología, pues ofrece una gama de posibilidades en el diseño y construcción de nuevos sistemas capaces de desarrollar diferentes funciones [3].

\section{La nanotecnología y la medicina}

Es bien conocido que a nivel biológico muchos de los procesos ocurren en el rango nanométrico, basta con saber que el ADN tiene un ancho de $2.5 \mathrm{~nm}$, una proteína típica entre 1 y $20 \mathrm{~nm}$ y la fuente de energía celular, el ATP, tiene una talla de $10 \mathrm{~nm}$. Algunos nanosistemas como los microtúbulos, el sistema de locomoción actina-miocina, los procesos de transporte celular, los ribosomas o incluso el ataque de un virus a una célula participan en mecanismos que ocurren justamente a esa escala [4]. La comprensión de muchos de estos procesos llevó, por un lado, a un mejor entendimiento del funcionamiento celular y en consecuencia de las enfermedades actuales, y, por otro, promovió la fabricación y diseño de nanoestructuras hechas por el hombre que puedan mimetizar o imitar las funciones de los sistemas naturales. Esto generó un nuevo enfoque para la investigación en las áreas químicobiológicas y de la salud, el cual tiene como meta llevar los tratamientos hacia una medicina más personalizada $[6,7]$. Dado que el tratamiento de algunas enfermedades tiene implicaciones éticas y socioeconómicas, las ideas sobre medicina para cada paciente se han venido asentando en conceptos como medicina genómica y vectorización (medicamentos con direccionamiento específico) 
para dirigir el sistema farmacéutico hacia el sitio de acción sin afectar otros órganos. Entonces, surge la nanomedicina, a la cual se le puede asignar como propósitos el seguimiento, control, construcción, reparación, defensa y mejoramiento de los sistemas biológicos humanos, trabajando desde el nivel molecular y utilizando la ingeniería de dispositivos y las nanoestructuras para lograr beneficios a la salud. Dicho de otra manera, la nanomedicina significa esencialmente la aplicación de la nanotecnología a la medicina. Las terapias conocidas hasta hoy como la cirugía, la radiación o la quimioterapia tienen como base eliminar los tejidos dañados en enfermos lo más rápido posible, mientras que el enfoque adoptado por la nanomedicina hace uso de las ventajas de los sistemas nanoestructurados para matar un grupo de células específicas, reparar tejidos mediante el uso de biosensores, diagnosticar enfermedades con sistemas de detección más sensibles, o bien controlar dónde, cuándo y cuánto fármaco debe ser liberado. En ese sentido, la propuesta de tratamientos mejorados se centra en poder evitar efectos secundarios, reducir las dosis frecuentes y desarrollar medicamentos eficaces con direccionamiento selectivo. Adicionalmente, la nanomedicina incluye el desarrollo de nanopartículas y superficies nanoestructuradas, así como técnicas para el diagnóstico molecular, tratamiento, seguimiento y terapia de enfermedades. Conceptos visionarios prevén la construcción y el control de las células artificiales mediante el uso de ingeniería de nanodispositivos y nanoestructuras para aplicaciones médicas [8].

\section{Nanotecnología y sistemas de liberación de fármacos}

La nanotecnología se ha aplicado con éxito en diferentes áreas como la ingeniería de tejidos, la biotecnología, la electrónica, los materiales y la administración de fármacos, y ha demostrado ser una herramienta útil para hacer frente a una variedad de desafíos que, en el caso de la tecnología farmacéutica y la administración de fármacos, están asociados con una baja biodisponibilidad, estabilidad y solubilidad, efectos secundarios no deseados, la entrega no específica, la poca eficacia terapéutica, niveles sanguíneos inconstantes y dosificación frecuente [7].

Las áreas de liberación y formulación de fármacos se han dirigido a diseñar sistemas a nivel submicrónico para combatir los desafíos anteriormente mencionados, con la finalidad de mejorar los tratamientos para las enfermedades actuales y ofrecer mejores alternativas. Estas nanoestructuras farmacéuticas han presentado múltiples ventajas: tienen la capacidad de proteger los fármacos encapsulados dentro de ellas contra la degradación hidrolítica y enzimática en el tracto gastrointestinal; dirigen la entrega de una gran cantidad de fármacos para diversas áreas del cuerpo; pueden sostener, disminuir o controlar la liberación; son capaces de suministrar fármacos, proteínas y genes a través de una ruta diferente a la administración oral; encapsulan y liberan fármacos muy hidrosolubles; previenen el efecto del primer paso 
FIGURA 1. a) Nanoacarreadores de primera generación; b) nanoacarreadores de segunda generación; c) nanoacarreadores de tercera generación.

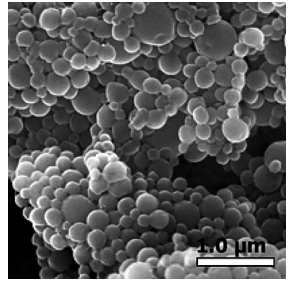

(a)

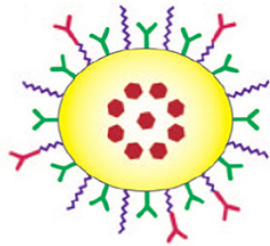

(b)

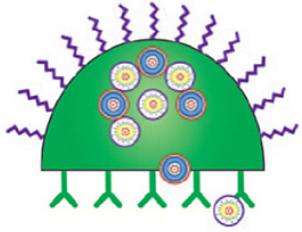

(c)

Fuente: Tomado de Riehemann et al. [8].

hepático, es decir, reducen el metabolismo de forma indirecta; aumentan la biodisponibilidad a través de proveer una mayor cantidad de fármaco en el lugar de absorción y de mantener por más tiempo el fármaco en el torrente sanguíneo; el controlar la liberación permite tener niveles sanguíneos constantes, minimiza las fluctuaciones y evita los efectos adversos o secundarios; $y$, finalmente, debido a su disminuido tamaño, son capaces de atravesar tejidos, ser absorbidos por células o bien presentar un efecto de retención en el sitio blanco [5]. El desarrollo de nanoestructuras para la liberación de fármacos, conocidos como nanoacarreadores, ha ido evolucionando con la nanotecnología y la biotecnología. A través de la manipulación de las propiedades de los materiales disponibles se han podido generar nanoestructuras con fines deseados, de esta forma el fármaco puede ser encapsulado, atrapado, adsorbido o unido químicamente a la nanoestructura [6]. Los nanoacarreadores actualmente empleados e investigados pueden ser clasificados en tres generaciones en función de las intenciones planteadas al momento de su desarrollo (figura 1).

La primera generación comprende un sistema de liberación pasivo que es localizado dentro del sitio blanco donde el contenido es liberado. Ejemplo de ello son las nanopartículas o liposomas, que cuando son administrados con la intención de alcanzar un tumor como tejido diana, se ven forzados a acumularse en el sitio de acción debido a un efecto de "permeación y retención promovida" o EPR, por sus siglas en inglés (figura 2). Este efecto se presenta dado el hecho de que los tumores presentan fenestraciones o interrupciones en la línea celular de los epitelios adyacentes a la vascularización. Los sistemas propuestos en esta generación se han modificado en su superficie con moléculas hidrofílicas como el polientilenglicol (PEG) con el objetivo de evitar su absorción por el sistema retículo endotelial y de esta forma aumentar su tiempo de residencia en sangre, los cuales son también conocidos como acarreadores furtivos o encubiertos "stealth". Los representantes de esta generación en uso clínico son los liposomas, nanopartículas de albumina (paclitaxel-Abraxane ${ }^{\circledR}$ ) y nanopartículas metálicas empleadas en 
FIGURA 2. La acumulación de nanopartículas a través del efecto EPR. 1) Las nanopartículas en circulación sanguínea; 2) extravasan a través de las fugas que presenta el epitelio adyacente al tumor; 3) hay una acumulación pasiva en el tumor.

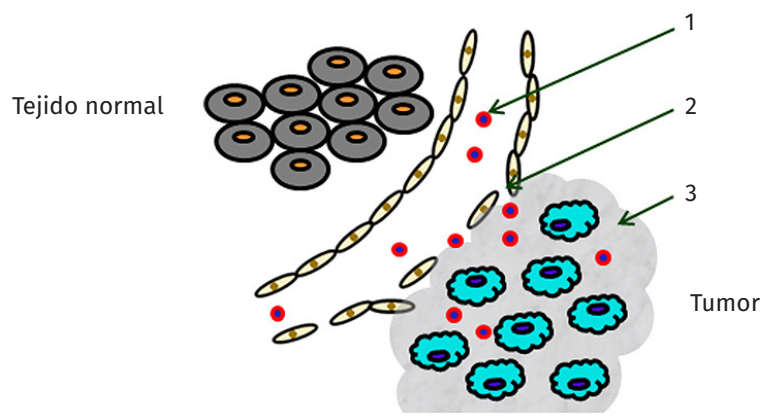

Fuente: Tomada de Wang et al. [23].

la diagnosis. En esta generación, la localización del acarreador en el sitio de acción sólo está dada por su tamaño.

La segunda generación abarca nanoacarreadores que han sido funcionalizados, modificados en su superficie, lo que les permite un reconocimiento molecular del sitio blanco. Por ejemplo, los liposomas o nanopartículas poliméricas funcionalizados con anticuerpos unidos a la superficie de la nanoestructura, para ser reconocidos por un grupo específico de células. También se han investigado otras moléculas para funcionalizar nanopartículas como son los nucleósidos, pequeños péptidos o marcadores moleculares que son expresados en el tejido de interés. En este grupo, también se incluyen sistemas que responden al ambiente, como los sistemas diseñados con materiales o polímeros sensibles al $\mathrm{pH}$, los que sufren una activación enzimática, o los que se activan por el estímulo de un agente externo como radiación, ultrasonido o radiofrecuencia (ejemplo, las nanopartículas de hierro que pueden ser controladas al someterse a un campo magnético). Esta generación se caracteriza por proponer sistemas para el tratamiento del cáncer principalmente; sin embargo, estos nanoacarreadores no han sido aún aprobados por la FDA.

Finalmente, los nanoacarreadores de la tercera generación están centrados en vencer las barreras biológicas de los órganos diana. Básicamente, los sistemas en este periodo son propuestos con múltiples funcionalidades, en otras palabras, combinando propiedades de las dos generaciones anteriores, centrándose en un diseño de sistemas capaces de realizar funciones más complejas como cruzar diferentes barreras biológicas y dirigirse hacia un determinado orgánulo intracelular [8].

\section{Algunos tipos de nanoacarreadores}

La nanotecnología en el área farmacéutica tiene como objetivo el desarrollo de sistemas con actividades individuales que se integren al funcionamiento 
del sistema biológico, o bien que puedan desarrollar funciones múltiples, es decir, que sean capaces de reconocer el órgano o tejido específico, por ejemplo, un grupo de células cancerígenas o bien que contengan algún tipo de marcador que avise de la existencia de este tejido. De esta forma se pretende que los nanosistemas propuestos reconozcan, diagnostiquen y participen en el tratamiento liberando el fármaco en el sitio de acción. Aún hay investigación para evaluar su eficiencia y existe un fuerte trabajo sobre las entidades regulatorias para que se obtengan sistemas económicos en producción, que sean controlables y cumplan con los requerimientos de calidad, eficacia y de seguridad necesarios.

Las tecnologías actuales hacen uso del conocimiento y manipulación de los materiales disponibles para generar sistemas avanzados de liberación de fármacos, de tal manera que, como se indicó anteriormente, estos sistemas han demostrado ser superiores a los sistemas convencionales de administración de fármacos [2]. Los materiales empleados determinan el tipo de nanoacarreador que se obtiene y éstos definen las propiedades y características de liberación de los fármacos que son incorporados en su interior. Se ha propuesto una amplia gama de nanoacarreadores que intentan cumplir las expectativas de los nuevos y mejorados tratamientos, entre los que se han incluido las nanopartículas poliméricas, los liposomas, las nanocápsulas, las nanoemulsiones, dendrímeros, etc. Características de algunos de ellos se describen a continuación.

\section{Nanopartículas poliméricas}

Son estructuras sólidas, con propiedades coloidales, generalmente esféricas, con un tamaño de 10 a $1000 \mathrm{~nm}$ (figura 3). El fármaco puede estar incorporado en su interior distribuido en la matriz polimérica, unido químicamente o adsorbido a la superficie de la nanopartícula. Son conocidas como

FIGURA 3. Nanopartículas de ácido poliláctico-co-glicólico (PLGA).

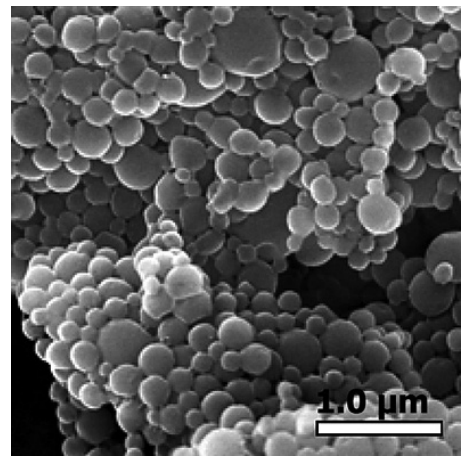

Fuente: Tomada de Freitas et al. [24]. 
nanoesferas o de tipo matriz, porque el fármaco está disperso molecularmente en toda la matriz. Ofrecen una liberación sostenida durante periodos más largos, por caso, días, semanas o meses. Pueden ser obtenidas empleando polímeros naturales o sintéticos, biodegradables o no. Su pequeña dimensión les permite penetrar en los capilares y ser absorbidas por las células, aumentando de este modo la acumulación de fármacos en los sitios de acción [9].

El tipo de polímero empleado en su manufactura determina su captación por las células. Por ejemplo, se ha observado que las nanopartículas fabricadas con policaprolactonas (PCL) son reconocidas más fácilmente por los macrófagos en comparación con aquellas que están hechas de ácido poliláctico-co-glicólico (PLGA) debido a las diferencias de hidrofobicidad en ambos materiales. Entre los materiales frecuentemente utilizados en la obtención de estas nanoestructuras se encuentra el quitosán, alginato, geltatina, poliacrilatos, policaprolactonas y poliésteres como el ácido láctico, el glicólico y copolímeros de estos como el ácido poliláctico-co-glicólico. La selección del material está relacionada con su biodegradabilidad y biocompatibilidad, pues se ha observado que algunos polímeros naturales generan respuesta inmune o productos de degradación que podrían provocar citotoxicidad. Otra ventaja de estos sistemas es que pueden ser reproducidos con buenos resultados, aún cuando la factibilidad de su escalado a nivel industrial sigue siendo un reto [10].

Varias estrategias se han empleado para dirigir estos sistemas hacia el órgano blanco. Se han ligado moléculas específicas como anticuerpos monoclonales, marcadores que son reconocidos por moléculas que se expresan en membranas de algunos tejidos como folatos que son reconocidos por receptores de folatos que se expresan en la mayoría de los tejidos cancerígenos. Otras aplicaciones que se han descrito incluyen la administración tópica para promover la permeación de fármacos a través de la piel o bien nanopartículas sensibles a pH que vierten su contenido a determinado $\mathrm{pH}$ para mejorar propiedades de biodisponibilidad de algunos fármacos.

\section{Liposomas}

Estas estructuras fueron los primeros nanoacarreadores empleados en la clínica. Se han investigado ampliamente desde 1970 como acarreadores de fármacos. Son estructuras vesiculares de entre 50 y $100 \mathrm{~nm}$ compuestas de fosfolípidos como la fosfatidilcolina, fosfatidilglicerol, fosfatidiletanolamina y fosfatidilserina. Una de las principales aplicaciones de estos sistemas es la administración de fármacos a través de la piel, permitiendo el paso de fármacos con peso molecular grande y alta solubilidad en agua. Se han propuesto para la administración pulmonar, por nebulizaciones, y también para la administración ocular. Presentan baja toxicidad debido a que los materiales 
FIGURA 4. Liposomas furtivos. Vesícula de fosfolípidos con fármaco encapsulado en su interior y cubierto con polietilenglicol (PFG).

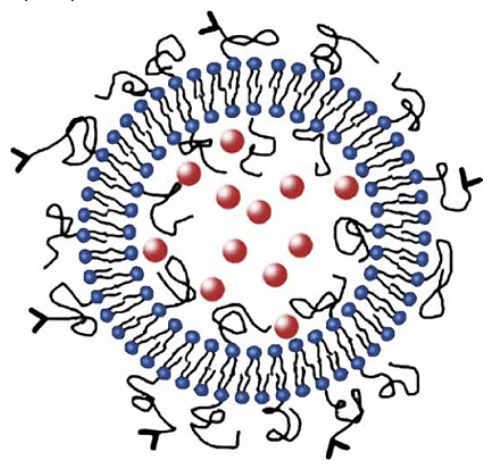

Fuente: Tomada de <http://openi.nlm.nih.gov/detailedresult.php>.

con que son obtenidos son moléculas endógenas que son bien aceptadas por el organismo, aunque esto, al mismo tiempo, representa una desventaja debido a que son reconocidos por el sistema retículo endotelial y son eliminados del cuerpo. La estrategia que se ha empleado para contrarrestar este problema ha sido la PEGilación, incorporación de polietilenglicol (PEG) en su superficie, lo cual permite obtener liposomas furtivos o encubiertos que ya no son reconocidos por este grupo de células, ver figura 4 [11].

Las propiedades fisicoquímicas y biológicas de los liposomas están determinadas por el tipo de lípidos con que son preparados, el material define la carga superficial, el tamaño e incluso el método de preparación. Los liposomas presentan algunas limitaciones como la baja eficiencia de encapsulación, se funden fácilmente en las membranas celulares liberando su contenido con antelación y durante su almacenamiento presentan fuga de fármaco, sin embargo, la modificación de la superficie o la incorporación de otros lípidos como el colesterol pueden conferirles estabilidad e integridad tras la administración oral o parenteral.

Se han descrito algunas modificaciones de estos nanoacarreadores y de acuerdo con sus nuevas propiedades se han identificado como transfersomas que incorporan un tensoactivo en su constitución, haciéndolos más flexibles sin llegar a desintegrase; etosomas como aquellos liposomas que tienen un alto contenido de alcohol, hasta un $45 \%$, y, niosomas que son producidos a partir de tensoactivos no iónicos [5].

\section{Nanopartículas sólidas lípidicas (SLN)}

Fueron introducidas en 1991 como sistemas acarreadores de fármacos y se preparan con lípidos como gliceril behenato, cetilpalmitato, glicerol tripalmitato. De forma general estos materiales son bien aceptados por el organismo, su biodegradabilidad hacen a este tipo de nanopartículas menos 
FIGURA 5. Nanopartículas sólidas lipídicas hechas de compritol (gliceril behenato) estabilizadas con Poloxámero 188, diámetro de $400 \mathrm{~nm}$.

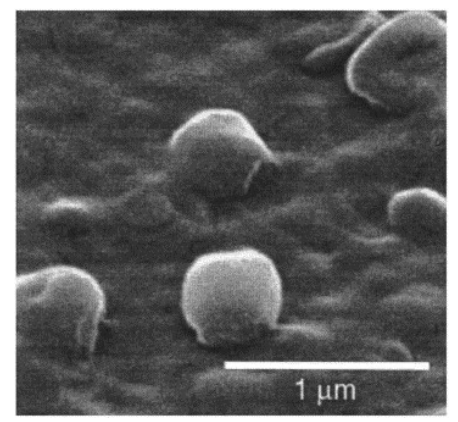

Fuente: Tomada de Müller et al. [12].

tóxicas que las poliméricas. Su forma generalmente es sólida y esférica (figura 5). Se obtienen en tallas entre 50 y $1000 \mathrm{~nm}$, proveen protección al fármaco contra la degradación y ofrecen una liberación sostenida. Nanopartículas lípidicas sólidas con tallas en el rango de 20 a 120 nm no son tomadas de forma rápida por el sistema retículo endotelial. Al tener los lípidos conjugados con el fármaco hacen factible la posibilidad de cargar tanto fármacos hidrofílicos como lipofílicos. Presentan buena reproducibilidad y se les puede asociar bajo costo debido a que en su obtención se emplean procesos de homogenización convencionales. Han sido propuestas para la administración oral, transdérmica, ocular y parenteral, pero se les ha atribuido la propiedad de atravesar la barrera hematoencefálica y alcanzar el tejido cerebral [12].

Una de sus posibles desventajas es que en sus procesos de obtención se utilizan altas temperaturas debido a que los lípidos deben ser fundidos, esto limita el tipo de fármaco que puede ser incorporado en estos sistemas. Presentan una baja eficiencia de encapsulación y se ha visto que expulsan el fármaco debido a un reacomodo de los lípidos durante su almacenamiento [13].

\section{Nanocápsulas}

Las nanocápsulas son nanopartículas poliméricas que contienen el fármaco en un núcleo líquido rodeado por una pared polimérica (ver figura 6), su tamaño se considera generalmente para radios que oscilan entre $100 \mathrm{y}$ $500 \mathrm{~nm}$. Varios enfoques técnicos se utilizan para la obtención de las nanocápsulas, sin embargo, los métodos comunes de preparación de nanocápsulas son polimerización interfacial, precipitación interfacial y los métodos de nanodeposición como nanoprecipitación, emulsión difusión, doble emulsión y coacervación, empleando polímeros preformados. Los parámetros más importantes a considerar en el diseño de una cápsula son el tamaño, el espesor de la membrana polimérica y su permeabilidad, así como su estabi- 
FIGURA 6. Nanoacarreadores.

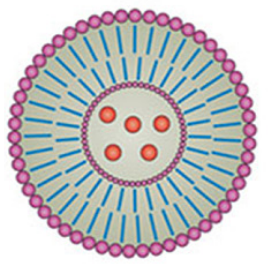

Liposomas

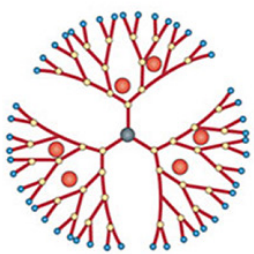

Dendrímeros

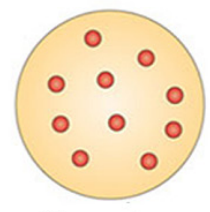

Nanoesferas

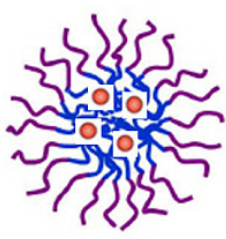

Micelas

Poliméricas

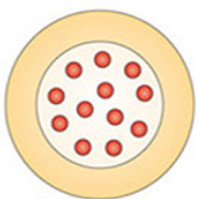

Nanocápsulas

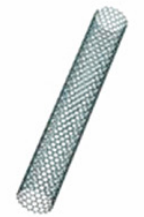

Nanotubos de Carbón

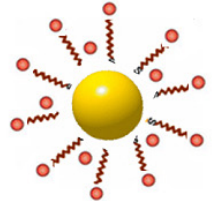

Nanopartículas de oro

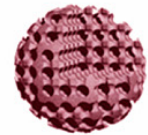
Nanopartículas de Óxido de Silicio

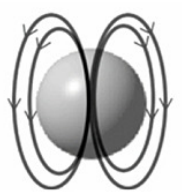

Nanopartículas Magnéticas

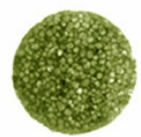

Nanopartículas de Fosfato de Calcio

O Fármaco

Fuente: Tomada de Orive et al. [25] y Ojea-Jiménez et al. [26].

lidad química. Estos sistemas han presentado gran interés, pues dependiendo del material de la capa protectora se pueden tener propiedades como control de la liberación de los fármacos [14].

\section{Dendrímeros}

Son nanoestructuras obtenidas empleando macromoléculas como la poliamidoamina (PAMAM), polipropilenimina y poliaril-éter. Son ampliamente ramificadas y con un núcleo interno. Su talla es generalmente menor a $10 \mathrm{~nm}$, pero pueden encontrarse entre 1 y $100 \mathrm{~nm}$ (figura 6). Los dendrímeros ofrecen ventajas como un menor índice de polidispersidad, múltiples sitios de unión, un tamaño bien definido que puede ser controlable y una estructura que puede ser fácilmente modificada para cambiar las propiedades químicas del sistema. Además, tienen una permeabilidad mejorada y efecto de retención que les permite llegar a las células tumorales con mayor eficacia que las moléculas pequeñas [15].

\section{Micelas poliméricas}

Son micelas esféricas formadas con varios copolímeros bloque anfifilícos (figura 6), usadas como vehículos para incrementar la solubilidad de fármacos hidrofóbicos, reducir la citotoxicidad y obtener liberación sostenida de fármacos. Micelas de óxido de polietileno (PEO), Poliestireno y Poli-isopro- 
pilacrilamina han sido ampliamente usadas para la encapsulación y liberación de fármacos. El núcleo interior de las micelas es hidrofóbico y generalmente está rodeado por una capa de polímero hidrofílico como el polietilenglicol (PEG). Debido a sus propiedades y materiales empleados se aumenta el tiempo de circulación en sangre y la acumulación del sistema en el sitio de interés. Dadas las propiedades de su núcleo se pueden incorporar fármacos liposolubles; sin embargo, la eficiencia de encapsulación está limitada al pequeño espacio contenido en el núcleo y la inestabilidad del fármaco en su interior provoca una liberación antes de llegar al sitio de acción. Se han empleado para la vectorización y liberación intracelular de fármacos [17]. La alta estabilidad estructural de las micelas poliméricas es una clave importante para la administración in vivo en forma micelar y elimina al mismo tiempo la posible contribución de una sola cadena de polímeros a la administración de fármacos, por lo tanto, aunque comparten la raíz de la palabra "micelas", las "micelas poliméricas" son muy diferentes a las micelas de bajo peso molecular formadas por tensoactivos [18].

\section{Nanoemulsiones}

Son emulsiones con un tamaño de gota entre 20 y $200 \mathrm{~nm}$ y con baja tensión interfacial agua/aceite. Son fáciles de preparar, generalmente de forma espontánea y en algunos casos sin la necesidad de incorporar energía externa. Se emplean para acarrear fármacos lipofílicos que sufren procesos de degradación por hidrólisis y como depósito inyectable en la administración subcutánea. Las ventajas observadas con estos sistemas son que aumentan la velocidad de absorción, ayudan a solubilizar fármacos liposolubles, pueden administrarse por varias rutas, aumentan la biodisponibilidad, son termodinámicamente estables, reducen las dosis frecuentes y los efectos adversos. Sus principales desventajas son la alta concentración de tensoactivos que pueden tener un efecto tóxico y su inestabilidad influenciada por cambios de $\mathrm{pH}$ y temperatura [16].

\section{Nanopartículas inorgánicas}

Nanopartículas de oro, plata, níquel, óxido de silicio y hierro (nanopartículas magnéticas), sulfato de calcio, fulerenos y nanotubos de carbono han sido propuestas para liberación intracelular de fármacos (figura 6). La superficie puede ser funcionalizada para promover el reconocimiento, permanencia y acumulación de estos sistemas en el órgano diana. Debido a que su talla oscila entre los 0.8 y $200 \mathrm{~nm}$, el área superficial se ve incrementada por lo que la incorporación de fármacos en la superficie permite cargar cantidades terapéuticas. Estas partículas inorgánicas presentan baja toxicidad. Nanopartículas de oro recubiertas con ácido oleico y polietilenimina han sido propuestas para la liberación intracelular en terapia génica, pues se ha visto que 
pueden atravesar la membrana nuclear. Los fulerenos y nanotubos de carbono presentan una buena biocompatibilidad, con propiedades químicas, eléctricas y ópticas que han sido aprovechadas para cargar en su superficie proteínas, péptidos y genes que después son liberadas en el interior de la célula. Mientras que nanopartículas de magnetita $\left(\mathrm{Fe}_{3} \mathrm{O}_{4}\right)$ han sido adsorbidas con fármacos y guiadas hacia el órgano blanco, por ejemplo, tumores, empleando un campo magnético externo que además las hace vibrar generando un aumento de temperatura que destruye las células malignas en un proceso conocido como termoablación [19].

\section{Sistemas ensamblados empleando el enfoque bottom-up con micro- y nanoestructuras para liberar fármacos sensibles}

Desde hace al menos tres décadas y gracias a los avances en biotecnología, macrobiomoléculas como péptidos, proteínas y análogos de ADN se han venido investigando como posibles agentes terapéuticos para el tratamiento de enfermedades como el cáncer o deficiencias genéticas [20]. El problema con estas moléculas es que presentan algunas limitaciones como fármacos, entre ellas su inestabilidad en fluidos biológicos y estrés fisicoquímico, lo que limita la ruta de administración y el tipo de acarreador a considerar. Las tecnologías descritas anteriormente, nanopartículas y liposomas han sido propuestas para encapsular este tipo de moléculas debido a que protegen el fármaco durante su administración y almacenamiento. Sin embargo, en el proceso de formulación convencional, la micro- y nanoencapsulación sufren estrés físico debido al contacto con disolventes orgánicos, interfaces por la generación de emulsiones, altas fuerzas de corte por homogenización, cambios de $\mathrm{pH}$ y altas temperaturas, provocando agregación, cambios conformacionales o degradación. Por ello, recientemente se han generado diferentes estrategias para evitar estos procesos como son el uso de polialcoholes, la adición de azúcares, el uso de ambientes no acuosos para evitar las interfaces agua/ aceite, reducción del tiempo de homogenización o bien agregar crioprotectores [21].

Conociendo estas desventajas de los procesos convencionales de nanoencapsulación, nuestro equipo de trabajo propuso una estrategia que asegura la integridad de los fármacos durante la etapa de formulación, la cual integra el concepto de infiltración de nanopartículas [28]. Dicha estrategia está basada en propiedades de adsorción de estas moléculas sobre la superficie de microesferas porosas y nanopartículas poliméricas. El aumento del área superficial asociado a la pequeña talla de las nanopartículas hace posible tener una gran superficie susceptible de ser empleada para adsorber cantidades terapéuticas de estos fármacos. Por otro lado, los sistemas porosos como las microesferas poliméricas tienen una gran área superficial debido a su alta porosidad y a los canales interconectados en su interior 
FIGURA 7. Sistemas ensamblados por adsorción/infiltración de nanopartículas. a) microesferas porosas; b) porosidad de la matriz interna de las microesferas porosas; c) y d) película de nanopartículas adsorbidas sobre la superficie de los poros de las microesferas.

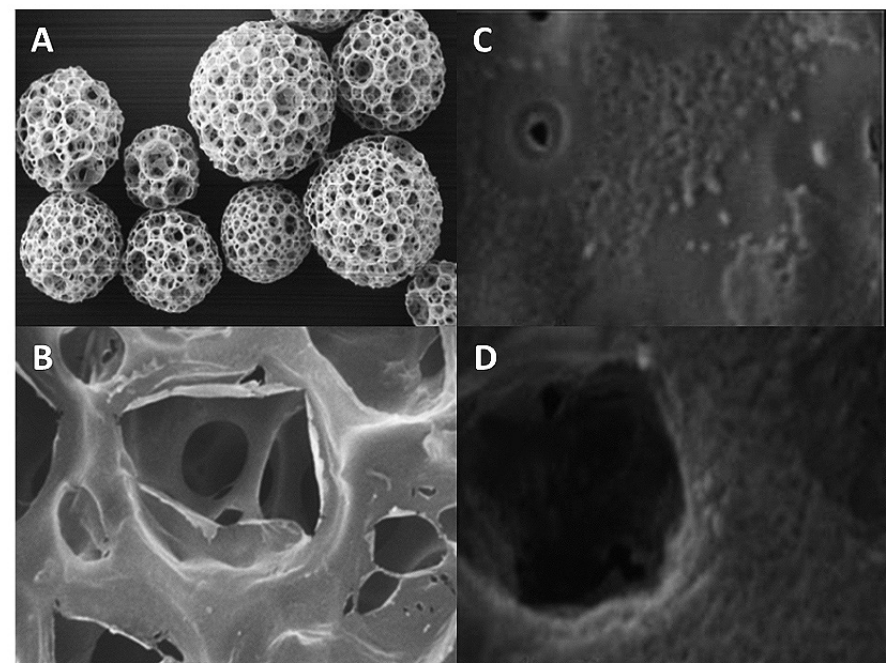

Fuente: Tomada de Alcalá-Alcalá et al. [27].

(figura 7). Así, en un primer paso las nanopartículas poliméricas son adsorbidas con el fármaco sensible, por ejemplo, péptidos y proteínas, y, posteriormente, son infiltradas al interior de microesferas porosas en un proceso simple de inmersión en medio acuoso con agitación suave. Este medio acuoso es un ambiente "amigable" para este tipo de moléculas, el cual también posee las condiciones fisicoquímicas apropiadas que favorezcan el proceso de adsorción, empleando el $\mathrm{pH}$ como parámetro a controlar pues tiene relación con puntos isoeléctricos que pueden provocar la generación de especies cargadas que pueden interaccionar con las propiedades de las superficies, por lo cual las fuerzas electrostáticas juegan un papel importante en el proceso de adsorción. Debido a que las microesferas porosas son sumergidas en la suspensión de nanopartículas, el fármaco puede estar adsorbido en ambas superficies (figura 8) [22].

Los resultados de estos sistemas ensamblados muestran que al aumentar la cantidad de nanopartículas presentes en la suspensión se aumenta la cantidad de fármaco que es incorporado al sistema; asimismo, la velocidad de liberación del fármaco se ve disminuida, creando la idea de una posible disminución en la frecuencia de administración. Se observó que estas nanopartículas infiltradas son adsorbidas a la superficie de las microesferas porosas formando una película que se vuelve cada vez más continua cuando la cantidad de nanopartículas aumenta (figura 8). Se ha sugerido que esta película de nanopartículas actúa como una barrera física que limita la difusión 
FIGURA 8. Proceso de obtención de sistemas ensamblados por adsorción/infiltración de nanopartículas biodegradables en microesferas porosas biodegradables para liberación de fármacos sensibles como péptidos y proteínas.

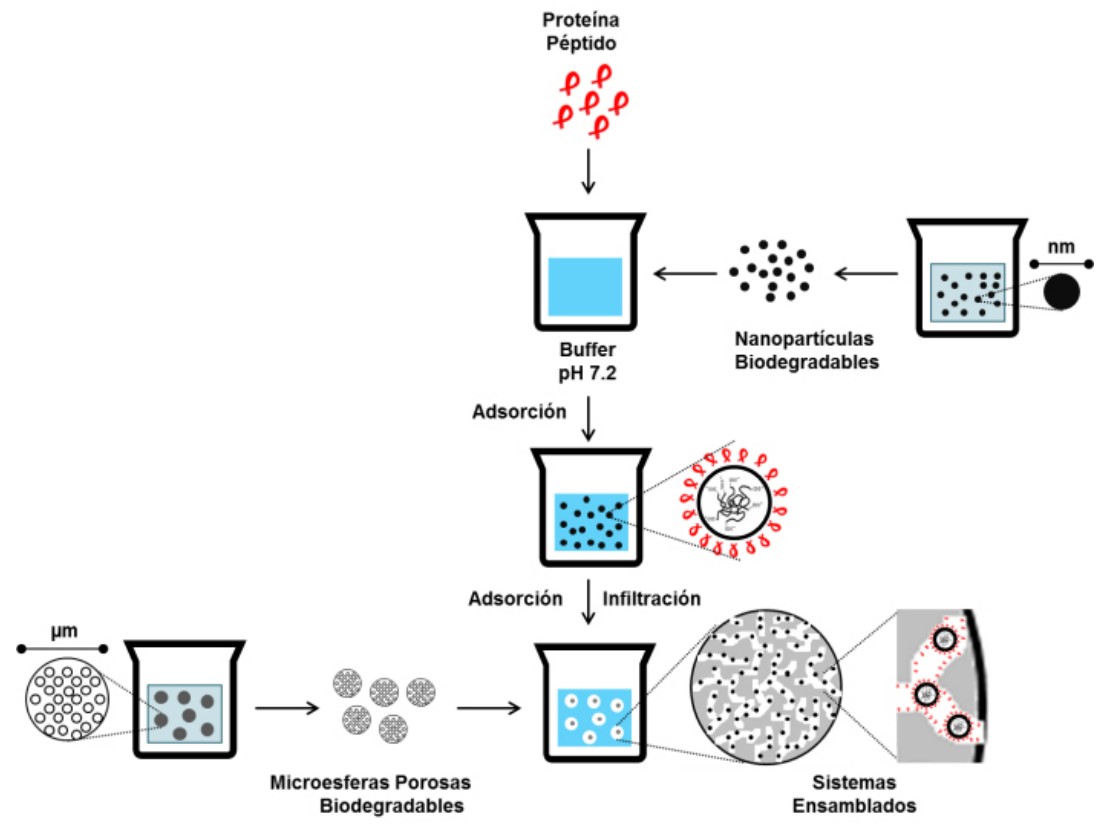

Fuente: Tomada de Alcalá-Alcalá et al. [27].

del fármaco desde el interior del sistema hacia el medio, por lo que el sistema sostiene la liberación o bien la controla en función de la cantidad de nanopartículas que satura el área superficial de la microesfera porosa. Los componentes del sistema, nanopartículas y microesferas porosas, son obtenidos en pasos por separado y sin la adición del fármaco en los procesos de obtención, por lo que los sistemas propuestos aseguran la integridad del fármaco durante su formulación pues se evitan las interfaces, contacto con solventes orgánicos y altas de fuerza de corte. Otras de las ventajas de estos sistemas ensamblados, bajo un enfoque de manufactura de "abajo hacia arriba", son su versatilidad para cargar diferentes fármacos sensibles en cantidades terapéuticas y la posibilidad de administrase por diferentes rutas, incluida la parenteral [27].

\section{Conclusiones}

El impacto de la nanotecnología en las ciencias de la salud radica en la necesidad de mejorar los tratamientos existentes para las enfermedades actuales, con la idea de poder administrar no sólo fármacos de bajo peso molecular sino también de fármacos biotecnológicos como péptidos, proteínas, 
enzimas o análogos de ADN. Formular todo tipo de fármacos en sistemas en la escala nanométrica presenta una serie de ventajas, indicadas anteriormente, pero, principalmente, se centran en dirigir y localizar la liberación de los fármacos en el sitio donde se desea que realicen su acción, modificando o funcionalizando su superficie. Gracias a su disminuida talla, estos sistemas son capaces de vencer diferentes barreras biológicas y llegar hasta el interior de las células. Estas propiedades se alcanzan controlando y manipulando las propiedades fisicoquímicas de los materiales.

El potencial de la nanotecnología para proveer beneficios a las áreas de la salud es enorme, pues se considera como una herramienta valiosa en el proceso de diseño y desarrollo de sistemas avanzados de liberación de fármacos. Aún existen muchos retos y áreas de oportunidad en el conocimiento de las enfermedades, su diagnosis y tratamiento. Mientras tanto, la nanotecnología farmacéutica va generando nuevos e interesantes retos en la liberación y administración de fármacos, aumentando la tendencia en la producción de trabajos de investigación, patentes, revisión y actualización de la regulación, con la meta principal de asegurar que los nuevos nanosistemas cumplan con los requerimientos de eficacia y seguridad para poder ser empleados en el área clínica.

\section{Referencias}

[1] Rocco, M. C. (2007) National Nanotechnology Initative. Handbook of nanosciences, Engeenering and Technology. USA: Taylor and Francis.

[2] Williams, D. (2008) The relationship between biomaterials and nanotechnology. Biomaterials, núm. 29: 1737-1738.

[3] Majumder, D. D., Banerjee, R., Ulrichs, C. H., Mewis, I. (2007) Nanomaterials: science of bottom-up and top-down. IETE Technical Review, núm. 24: 9-25.

[4] Ochekpe, N.A., Olorunfemi, P.O., Ngwuluka, N.C. (2009) Nanotechnology and drug delivery, part 1: Background and applications. Tropical Journal of Pharmaceutical Research, vol. 8, núm. 3: 265-274.

[5] Ochekpe, P.O., Olorunfemi, N.A., Ngwuluka, N.C. (2009) Nanotechnology and drug delivery, part 2: Nanostructures for drug delivery. Tropical Journal of Pharmaceutical Research, vol. 8, núm. 3: 275-287.

[6] Ruenraroengsak, P., Cook, J.M., Florence, A.T. (2010) Nanosystem drug targeting: Facing up to complex realities. Journal of Controlled Release, núm. 141: 265-276.

[7] Roco, M.C. (2003) Nanotechnology: Convergence with modern biology and medicine. Current Opinion in Biotechnology, núm. 14: 337-346.

[8] Riehemann, K., Schneider, S.W., Luger, T.A., Godin, B., Ferrari, M., Fuchs, H. (2009) Nanomedicine-Challenge and perspectives. Angewandte Chemie International Edition, núm. 48: 872-897.

[9] Singh, R., Lillard Jr., J.W. (2009) Nanoparticle-based targeted drug delivery. Experimental and Molecular Pathology, vol. 86, núm. 3: 215-223. 
[10] Chan, J.M., Valencia, P.M., Zhang, L., Langer R., Farokhzad, O.C. (2010) Polymeric nanoparticles for drug delivery. Methods in Molecular Biology, núm. 624: 163-175.

[11] Kulkarni, P. Yadav, J.D., Vaidya, K. (2010) Liposomes: A novel drug delivery system. International Journal of Current Pharmaceutical Research, vol. 3, núm. 2: 201.

[12] Müller, R.H., Mäder, K., Gohla, S. (2000) Solid lipid nanoparticles (SLN) for controlled drug delivery - A review of the state of the art. European Journal of Pharmaceutics and Biopharmaceutics, vol. 5, núm. 2: 161-177.

[13] Waghmare, A.S., Grampurohit, N.D., Gadhave, M.V., Gaikwad, D.D., Jadhav, S.L. (2012) Solid lipid nanoparticles: A promising drug delivery system. International Research Journal of Pharmacy, vol. 3, núm. 4: 100-107.

[14] Mayer, C. (2005) Nanocapsules as drug delivery systems. International Journal of Artificial Organs, vol. 28, núm. 11: 1163-1171.

[15] Malik, A., Chaudhary, S., Garg, G., Tomar, A. (2012) Dendrimers: A tool for drug delivery. Advances in Biological Research, vol. 6, núm. 4: 165-169.

[16] Devarajan, V., Ravichandran, V. (2011) Nanoemulsions: As modified drug delivery tool. Pharmacie Globale Iinternational Journal of Comprehensive Pharmacy, vol. 2, núm 4: 1-6.

[17] Jones, M.C., Leroux, J.C. (1999) Polymeric micelles - A new generation of colloidal drug carriers. European Journal of Pharmaceutics and Biopharmaceutics, núm. 48: 101-111.

[18] Miyata, K., Christie, R. J., Kataoka, K. (2011) Polymeric micelles for nanoscale drug delivery. Reactive and Functional Polymers, vol. 71, núm. 3: 227-234.

[19] Xu, Z.P., Zeng, Q.H., Lu, G.Q., Yu, A.B. (2006) Inorganic nanoparticles as carriers for efficient cellular delivery. Chemical Engeenering Science, vol. 61, núm. 3: 1027-1040.

[20] Saez V., Hernández J.R., Peniche C. (2007) Microspheres as delivery systems for the controlled release of peptides and proteins. Biotechnoly and Applied Biochemistry, vol. 24: 98-107.

[21] Van de Weert, M., Hennink, W.E., Jiskoot, W. (2000) Protein instability in poly(lactic-co-glycolic acid) microparticles. Pharmaceutical Research, núm. 17: 1159-1167.

[22] Alcalá, S. (2010) Encapsulación de acetato de leuprolide por infiltración de nanopartículas biodegradables en microesferas biodegradables porosas. México: UNAM.

[23] Wang T., Upponi, J.R., Torchilin, V.P. (2012) Design of multifunctional non-viral gene vectors to overcome physiological barriers: Dilemmas and strategies. International Journal of Pharmaceutics, vol. 427, núm. 1: 3-20.

[24] Freitas, S., Hielscher, G., Merkle, H.P., Gander, B. (2004) A fast and simple method for producing biodegradable nanospheres. European Cells and Materials, vol. 7, núm 2: 28.

[25] Orive, G., Anitua, E., Pedraz, J.L., Emerich, D.F. (2009) Biomaterials for promoting brain protection, repair and regeneration. Nature Reviews Neuroscience, núm. 10: 682-692. 
[26] Ojea-Jiménez, I., Tort, O., Lorenzo, J., Puntes, V.F. (2012) Engineered nonviral nanocarriers for intracellular gene delivery applications. Biomedical Materials, vol. 7, núm. 5: 1-13.

[27] Alcalá-Alcalá, S., Urbán-Morlán, Z., Aguilar-Rosas, I., Quintanar-Guerrero, D. (2013) A biodegradable polymeric system for peptide-protein delivery assembled with porous microspheres and nanoparticles using an adsorption/infiltration process. International Journal of Nanomedicine (en prensa).

[28] Rodríguez-Cruz, I.M., Domínguez-Delgado, C.L., Escobar-Chávez, J.J., LeyvaGómez, G., Ganem-Quintanar, A., Quintanar-Guerrero, D. (2009) Nanoparticle infiltration to prepare solvent-free controlled drug delivery systems. International Journal of Pharmaceutics, núm. 371: 177-181. 